\title{
Human-Centric Functional Modeling and Defining Metrics for the Function and Level of Consciousness
}

Andy E. Williams, Nobeah Foundation, Nairobi, Kenya

\begin{abstract}
Human-Centric Functional Modeling provides a methodology for defining the properties of systems. This short paper explores its application to defining a representation of, and metrics for, both impaired consciousness as well as the heightened levels of consciousness sometimes referred to as "enlightenment" in various traditions.
\end{abstract}

\section{Introduction}

Human-Centric Functional Modeling [1] hypothesizes that any system can be understood in terms of the set of functions it can be observed by humans to have, and in terms of the different states the system might occupy as a result of these functions, where these states are "functional states" because they are defined in terms of the functions available to transition into those states or out of those states. From this perspective, as the system acts by executing these functions or by having these functions executed on it, the system moves through a space of functional states or a "functional state space".

Use of this functional state space to represent all functions of cognition, even where the mechanisms through which those functions are implemented are unknown, enables mathematical expressions to be used to define properties of cognition. This enables analogies to be drawn with these mathematical expressions so that all equivalent properties in other systems are intuitively understandable through comparison with properties of cognition. For example, in the conceptual space that represents the functional state space of the cognitive system, and in the collective conceptual space that represents the functional state space of the collective cognition, general problem-solving ability is hypothesized to be the volume of conceptual space that can be navigated by the cognition per unit time, multiplied by the density of concepts in that volume which the cognition has to move through. As an example of this representation applying even where the mechanisms through which those functions are implemented are unknown, we might have a functional model for the reasoning connecting the concept of a mouse to the concept of a horse, where that reasoning determines the size of the mouse to be less than that of the horse. However, we might lack any model whatsoever of the structures in the brain through which that reasoning is executed. Unlike other approaches [2], this completely functional approach helps avoid any need to understand the neural correlates of consciousness whatsoever [3], [4], but also provides a model for fully incorporating such information when it becomes available.

Human-Centric Functional Modeling defines a universal approach for modeling the behavior of all systems in terms of motion through "functional state spaces" similar to the functional state space the human cognitive system can be represented as navigating. In the case of consciousness, the consciousness system is represented as navigating a space of awareness's (an "awareness space").

\section{Functions of Consciousness}

In Human-Centric Functional Modeling there are two sets of functionality related to consciousness. The external (consciously observable) functionality is represented in terms of paths through the functional state space (the awareness space) of the consciousness system. The internal functionality is represented as the functionality required for the consciousness system to exhibit the external functionality. This internal functionality is represented as the six functions hypothesized to be required for the consciousness system to navigate the awareness space with general problem-solving ability, and therefore with the ability to solve any problem of consciousness in general. As mentioned, this general 
problem-solving ability has been represented in terms of dynamics that are stable within some fitness space, so the system maintains its fitness to execute all of its functions and is able to potentially persist in any problem-solving effort until the problem is solved.

From this HCFM perspective, functions that are commonly measured as part of assessing the level of ability of a consciousness to function, are external (consciously observable), and therefore should each map to a path through the awareness space. Any test of impaired consciousness [5] is then hypothesized to be represented by a series of exercises which attempt to navigate the consciousness through a set of such paths, where failure of that element of the test is represented by the inability of the consciousness to perform that navigation. As an example, assessment of disorders of consciousness after a stroke might performed by the Glasgow Coma Scale [6] and the Diagnostic and Statistical Manual of Mental Disorders [7], where the severity of the stroke itself might be determined through the National Institutes of Health Stroke Scale [8]. Some of the states of consciousness defined in the Glasgow Coma Scale are coma, stupor, and somnolence. From this HCFM perspective, each of these states has a specific representation in terms of the functions of awareness that can and cannot be executed from them. Since each function of awareness is represented as a process that moves awareness from one experience or awareness state to another, this inability to execute such awareness processes implies the consciousness is potentially unable to navigate to certain regions of awareness space. Interpretation of the various measures of consciousness in relation to conditions such as stroke [9], [10], [11] in terms of this functional model, as well as clinical validation of these interpretations, remains to be pursued.

\section{Enlightenment and Level of Consciousness}

Where general problem-solving ability or intelligence is hypothesized to be the volume of conceptual space that can be navigated by the cognition per unit time multiplied by the density of concepts in that volume which the cognition has to move through, by analogy, in Human-Centric Functional Modeling the individual's level of enlightenment [12] is hypothesized to be the general problem-solving ability of the consciousness, that is, the ability of the consciousness to navigate to an awareness which solves the problem of conscious well-being. This is hypothesized to be the volume of awareness space (which also might be referred to as the consciousness or experiential space) that can be navigated by the consciousness per unit time, multiplied by the density of experiential states in that volume which the consciousness has to move through.

Understanding the difference between consciousness and cognition in this model has critical implications in the field of psychology and in treating conditions like depression. Cognition from this perspective is like a calculator that navigates from one concept to another as it executes its calculations. Cognition sees everything as a conceptual problem. Consciousness sees everything as a problem of awareness that might not even have a conceptual representation at all. In other words, from the perspective of HCFM, achieving conscious well-being is not a problem of cognition, it is a problem of where one places one's awareness. As a concrete example, the cognition might try to understand whether the glass is half empty or half full by engaging in ever more complex and precise calculations of whether the glass has greater than or less than $50 \%$ of its volume filled. Or the consciousness might just direct its awareness to the part of the glass that is half full. The mind is only one of the systems which the consciousness might direct its awareness to. But since complex problems of cognition can't reliably be solved, then relying on cognitive problem-solving to create the ability of the consciousness to reliably direct itself to the part of the glass that is half full would not seem a reliable strategy towards conscious well-being. This suggests that one potentially interesting area of study might be exploring whether practices which elevate consciousness might be more effective than the attempting to rely on cognitive processes in achieving a patient's psychological well-being, in which case leveraging such 
cognitive processes in attempting to elevate a patient's capacity for self-analysis might not be as effective when it comes to reliability in achieving a patient's psychological well-being.

It should be noted however that although this awareness space has been defined conceptually, and although that conceptual representation might be extremely useful in understanding the functions of consciousness and in understanding properties like "enlightenment", no clinical validation of this concept of an "awareness space", and no clinical measurement of any individual's actual awareness space yet exists. As of this writing, a representation of functional state space has only been approximated. The underlying challenge is that there are a number of elements that must be elaborated before a complete representation of the graph of any functional state space is possible, whether talking about the conceptual space, the awareness space, or the functional state space of any other system. One of the missing elements is how to quantify distances in functional state space. Though distances have been approximated, they have not been precisely defined. However, functional state spaces can potentially be used to represent a very wide range of systems, including virtually any biological system [13], and in addition can potentially be used to represent any physical system up to and including the entire physical universe [14]. Solving the representation problem for any functional state space is predicted to solve it for all functional state spaces. Because defining functional state spaces for any given system is predicted to vastly and perhaps even exponentially increase [16] our collective ability to solve any problem in general concerning that system, there is then great incentive for the entire scientific community, from physicists to biologists, to psychologists, to mathematicians, to solve this representation problem.

How does this enlightenment in functional state space compare to enlightenment in the sense of the word commonly found in existential traditions such as the yogic tradition or Buddhism? HumanCentric Functional Modeling hypothesizes that while moving through its functional state space consciousness or any other living system also takes moves through some "fitness space" that describes its fitness to execute all of its functions. If the system has a stable set of functionality, it is then expected that it will stay within a bounded region within this space.

The doctrine of non-entanglement with the world has been described as an important part of the path to enlightenment [15]. Non-entanglement has sometimes been described as non-attachment, but some teachers in the yogic tradition such as the individual known as "Sadhguru" have distinguished the two, with attachment being said to be positive and entanglement as being said to have a negative impact on capacity for well-being. One metaphor illustrating the difference is to imagine sailing to an undiscovered tropical island paradise visible in the distance. When one arrives, one drops anchor to enjoy the island. In this way all attachments are anchors that add to the richness of life. But on the other hand, the rope attached to that heavy anchor might wrap around one's leg, and when the anchor goes over board it might drag one to the bottom against one's will if one is not able to escape. In this way all entanglements are anchors that harm our well-being wherever we are unable to release them. In terms of this functional model, entanglements are awareness's for which we lack the awareness processes to reliably navigate away from.

In the case of Buddhism, the account of the Budda's enlightenment has been described as discovering a position "at the center of a wheel" in which one might remain at peace despite the turning of events in the world. Human-Centric Functional Modeling hypothesizes that the path that consciousness or any other living system takes through functional state space is dynamically stable in the "fitness space" of the system, where the dynamics in fitness space achieve that stability through some cyclic pattern of motion. The dynamics of the consciousness as a system moving through its own functional state space (the consciousness or awareness space) while maintaining stability in its own fitness space is then 
potentially represented by a cyclic pattern, or metaphorically a "wheel". This wheel is defined in terms of a stability between predicted, targeted, and actual values of fitness where this stability is unconscious. This interpretation remains to be confirmed clinically. Furthermore, the theoretical model itself also requires elaboration. For example, through various Buddhist practices it is said to be possible to make this stability conscious, what does this mean in the hypothetical functional state space and fitness space of the consciousness system? Does making this stability conscious make it possible to radically expand the wheel so that one is undisturbed by a far greater proportion of events?

\section{The Interaction Between Cognition and Consciousness}

In this model of cognition, outcomes are selected according to impact on fitness in executing all cognitive functions, which is a functional representation of well-being of the cognitive system. The individual cognition might be represented as reasoning (selecting reasoning processes to execute) according to impact on cognitive well-being, therefore the difference between outcomes of reasoning and impact on cognitive well-being is important. Both the cognitive system and the consciousness system have some level of fitness to execute all of their functions, hence both have their own metric for well-being (cognitive well-being in the case of the cognitive system and conscious well-being in the case of the consciousness system). An individual's perception of their well-being is hypothesized to be represented by their conscious well-being to the degree that they are not "identified" with the mind, and therefore don't confuse their mind with "themselves".

Individuals however vary widely in their knowledge of what impacts their conscious well-being. In this cognitive model, the general accuracy in the individual's understanding of what impacts conscious well-being, and therefore the individual's ability to reliably achieve and conscious maintain well-being, is referred to as mentioned as the individual's degree of "enlightenment". In terms of the interaction between the cognitive system and the consciousness, increasing the level of one's consciousness in one's reasoning processes has the objective and well-defined meaning of being an increase in capacity to navigate one's conscious awareness so that navigating a larger volume "fitness space" of the cognitive system per unit time, so that in turn navigating to greater cognitive fitness is reliably achievable. In other words, a higher level of consciousness is predicted to result in the ability to see problems in the simplest way possible, as well as the ability to use the simplest and most effective reasoning to solve much more difficult problems where doing so is beneficial.

Defining cognitive enlightenment (as opposed to the enlightenment of consciousness described above) as the capacity to navigate the conceptual space in order to reliably achieve well-being, then the individual's degree of cognitive enlightenment is also related to the individual's cognitive assessment of what is better. HCFM defines all concepts as belonging to a "conceptual space" and defines all cognitive processes as navigating between those concepts (those points in conceptual space). A greater degree of cognitive enlightenment implies a greater understanding of how a larger range of things (concepts in conceptual space) are related to the entity's conscious well-being. And an understanding of how a larger range of things are related to the entity's well-being is in effect expanding the entity's consciousness of what the boundaries to itself actually are. For example, an individual might be conscious that the quality of the water and air around them is related to their conscious well-being. In this sense, the air and the water are part of their conscious well-being as well.

In this sense, the degree of conscious enlightenment represents the degree to which the individual has expanded their consciousness of what the boundaries to their individual self actually are, so a greater impact on individual well-being is possible. As an example, if an individual only sees a full stomach as well-being, then they might miss out on actions that achieve anything else, which is virtually every action. If an individual only sees their personal condition as being related to their well-being, then they 
miss out on actions that give them the gift of being of service to others. When the sense of self has been enlarged, then the individual will look for impact on well-being rather than on a specific outcome. And when the understanding of what impacts well-being is enlarged, the individual might look for impact on well-being for others rather than for this individual physical body, this individual emotional system, or this individual mind alone. This and every other definition of any property of consciousness discussed in this paper remains to be validated. The point is not to claim this validity has been confirmed, only to demonstrate that a self-consistent representation of potentially every property of consciousness can be defined in this hypothetical model.

\section{Conclusions}

Any discussion of consciousness and its functions might benefit from being based on a well-defined functional model of consciousness. Without such a model, the words describing sufficiently complicated ideas about consciousness can be parroted but it might be that an understanding of any such words can't reliably be exchanged. This paper has proposed one functional model of consciousness that might be used to define a scale for consciousness that is analogous to the scale measuring each individual's relative intelligence quotient in terms of a $\mathrm{g}$ factor, and has attempted to validate that the model can at least be used to conceptualize a representation for the properties of consciousness measured by some existing clinical tests of consciousness.

\section{References}

[1] Andy E. Williams, A Revolution in Systems Thinking?, Proceedings 2021 Congress of the World Organization of Systems and Cybernetics (WOSC), in press (2022)

[2] Gamez, David. "The measurement of consciousness: a framework for the scientific study of consciousness." Frontiers in Psychology 5 (2014): 714.

[3] Chalmers, D. (2000). "What is a neural correlate of consciousness?," in Neural Correlates of Consciousness, ed T. Metzinger (Cambridge, MA: MIT Press), 17-39.

[4] Aru, J., Bachmann, T., Singer, W., and Melloni, L. (2012). Distilling the neural correlates of consciousness. Neurosci. Biobehav. Rev. 36, 737-746. doi: 10.1016/j.neubiorev.2011.12.003

[5] Wijdicks EFM, Cranford RE. Clinical diagnosis of prolonged states of impaired consciousness in adults. Mayo Clinic Proceedings. 2005;80(8):1037-1046.

[6] Teasdale G, Jennett B. Assessment of coma and impaired consciousness. A practical scale. The Lancet. 1974;2(7872):81-84.

[7] Anonymous. Diagnostic and Statistical Manual of Mental Disorders. 4th edition. Washington, DC, USA: American Psychiatric Association; 2000.

[8] Lyden PD, Lu M, Levine SR, Brott TG, Broderick J. A modified national institutes of health stroke scale for use in stroke clinical trials preliminary reliability and validity. Stroke. 2001;32(6):1310-1316.

[9] Weir CJ, Bradford APJ, Lees KR. The prognostic value of the components of the Glasgow Coma Scale following acute stroke. Monthly Journal of the Association of Physicians. 2003;96(1):67-74.

[10] Gustafson Y, Olsson T, Erikkson S, Asplund K, Bucht G. Acute confusional states (delirium) in stroke patients. Cerebrovascular Diseases. 1991;1(5):257-264. 
[11] Hénon H, Lebert F, Durieu I, et al. Confusional state in stroke: relation to preexisting dementia, patient characteristics, and outcome. Stroke. 1999;30(4):773-779.

[12] Costeines, M. (2009). What enlightenment means: A qualitative study of nondual consciousness as experienced by teachers of nondual mysticism. Institute of Transpersonal Psychology.

[13] Williams, A. E. (2022, February 1). Modeling Biological Systems Using Functional State Spaces. https://doi.org/10.31730/osf.io/6k4z5

[14] Williams, A. E. (2022, February 1). The Importance of Representing the Physical Universe or Virtual Universes in Functional State Space to the Progress of Physics.

https://doi.org/10.31730/osf.io/54qrz

[15] Amaro, Ajahn. "Guided Sitting and Walking Meditations on Emotion." Mindfulness 10.6 (2019): 1186-1187.

[16] Williams, A. E. (2020, July 11). Human Intelligence and General Collective Intelligence as Phase Changes in Animal Intelligence. https://doi.org/10.31234/osf.io/dr8qn 